\title{
A CHR-based Implementation of Known Arc-Consistency
}

\author{
MARCO ALBERTI, MARCO GAVANELLI, EVELINA LAMMA \\ Dipartimento di Ingegneria, Università degli Studi di Ferrara \\ PAOLA MELLO, MICHELA MILANO \\ Dipartimento di Elettronica, Informatica e Sistemistica, Università degli Studi di Bologna \\ submitted 31 August 2002; revised 13 November 2003, 30 June 2004; accepted 9 August 2004
}

\begin{abstract}
In classical CLP $(F D)$ systems, domains of variables are completely known at the beginning of the constraint propagation process. However, in systems interacting with an external environment, acquiring the whole domains of variables before the beginning of constraint propagation may cause waste of computation time, or even obsolescence of the acquired data at the time of use.

For such cases, the Interactive Constraint Satisfaction Problem (ICSP) model has been proposed (Cucchiara et al. 1999a) as an extension of the CSP model, to make it possible to start constraint propagation even when domains are not fully known, performing acquisition of domain elements only when necessary, and without the need for restarting the propagation after every acquisition.

In this paper, we show how a solver for the two sorted CLP language, defined in previous work (Gavanelli et al. 2004) to express ICSPs, has been implemented in the Constraint Handling Rules (CHR) language, a declarative language particularly suitable for high level implementation of constraint solvers.
\end{abstract}

\section{Introduction}

Constraint Logic Programming on Finite Domains (CLP $(F D)$ ) represents one of the most successful implementations of declarative languages. By means of constraints, the user can give the specifications of a combinatorial problem and possibly solve it, exploiting efficient propagation algorithms. CLP $(F D)$ languages have been successfully used for solving a variety of industrial and academic problems. However, in some constraint problems, where domain elements need to be acquired, it may not be wise to perform the acquisition of the whole domains of variables before the beginning of the constraint propagation process. For instance, in configuration problems (Mailharro 1998 ILOG 1999) domain elements represent components, which have to be synthesized before being used. The set of components is not known beforehand, and sometimes even the size of the set cannot be estimated. Often, a minimization of the set of components is required, thus the constraint solver produces a new component only when it is strictly necessary.

In systems that need to interact with an external environment, domain elements 
can be produced by an acquisition system that retrieves information about the outer world. An example is given by Faltings and Macho-Gonzalez (2003) where Internet applications are faced and obviously not all the information can be computed before starting the constraint satisfaction process. As another example, consider a visual search system (Cucchiara et al. 1999b) where domain elements are basic visual features (like segments, points, or surface patches) extracted from the image. In a classical CLP $(F D)$ computation, all domain values must be known when defining the variables, so all the possible visual features would have to be extracted before starting the visual search process, even if only a small subset of them will be actually used. The synthesis of visual features is usually very time consuming, because the information encoded with signals must be converted into symbolic form. Thus, the extraction of domain elements that will not be used can result in a significant waste of computation time. Also, in systems that interact with an evolving environment, full acquisition of all the domain elements is not wise (Barruffi et al. 1999). In fact, if all the possible information is acquired beforehand, some of the information might be obsolete at the end of the acquisition.

For all these reasons, a new model called Interactive Constraint Satisfaction Problem (ICSP) has been proposed (Cucchiara et al. 1999a) as an extension of the widely used Constraint Satisfaction Problem (CSP) model. In an ICSP, domains consist of a known part, containing the available elements, plus a variable that semantically represents a set of values that could be added to the domain in the future. In a sense, in an ICSP, domains can be considered as streams of information from one system to the constraint solver. Constraint propagation can be performed even when the domains are not completely known, and domain values can be requested from an acquisition system during constraint propagation; in other words, constraint propagation and value acquisition interact (thus Interactive in the name of the framework) and are interleaved, whereas in classical CSP frameworks domain elements are completely known before the beginning of the propagation. In this way, the acquisition system can possibly extract only elements consistent with the imposed constraints, thus focusing the attention only on significant data. Various propagation algorithms have been proposed (Cucchiara et al. 2001) for exploiting the available information and acquiring new domain values only when strictly necessary. Reducing the number of extracted elements can provide a notable speedup (Cucchiara et al. 1999a).

In (Gavanelli et al. 2004) we describe a corresponding CLP language. Our language is two sorted. The first sort is the classical sort on Finite Domains $(F D)$. The second sort, called $\mathcal{I}$-Set, is based on a structure similar to streams, and represents domains of the $F D$ variables. From the constraints on the $F D$ sort, the system can start propagation before having full knowledge of domain elements. Each element will be inserted on demand in the domain, without having to restart constraint propagation from scratch. Moreover, constraints can be imposed on domains, thus helping the user defining I-Sets ${ }^{1}$ declaratively. In this paper, we present an imple-

1 In this article, the $\mathcal{I}$-Set notation (with calligraphic $\mathcal{I}$ ) will denote the sort, while the notation I-Set (with non-calligraphic I) will denote a particular I-Set. 
mentation of the two-sorted language in Constraint Handling Rules (CHR). CHR (Frühwirth 1998) is a declarative language for defining new constraint solvers at a very high level. CHR can be used for rapid prototyping, and has proven effective in various real life applications. The purpose of this paper is to show how Constraint Handling Rules can be effectively used to implement the solver for our two sorted ICSP-based language. In previous work (Cucchiara et al. 1999a), the propagation algorithms have been proposed and separately implemented, but we did not describe the full implementation of the solver for ICSP problems. The algorithms were implemented using non fully declarative constructs (e.g., metaterms with destructive assignment). The high level, declarative encoding in CHR consists of a solver for the $\mathcal{I}$-Set sort, a solver for the $F D$ sort, and an interface between them designed to exploit the advantages of the ICSP model in systems interacting with external acquisition modules.

Of course, other aspects in the model of a problem, besides domain elements, could be unknown: in a CSP there could be unknown variables or unknown constraints. Typically, in all Constraint Programming systems new constraints can be easily added, while removal of constraints is more complex (Dechter and Dechter 1988). The addition of variables has been taken into account by Dynamic CSP models (Mittal and Falkenhainer 1990). Our work is focussed on unknown domain elements, and proposes an interaction based on the acquisition, from an external system, of domain elements.

The rest of the paper is organized as follows. The declarative and operational semantics of the language defined by Gavanelli et al. (2004) are briefly recalled in Section [2] In Section [3 we describe the architecture of the language from an implementation viewpoint, and in Section 4 we show how it is implemented in the CHR language. Discussion of related work (including a detailed comparison with (Mailharro 1998), which is very related to our work from the operational viewpoint) and conclusions follow.

\section{Syntax and Semantics}

The language defined in (Gavanelli et al. 2004) is based on a two sorted CLP, where the first sort is the classical sort on Finite Domains $(F D)$ and the second is the sort on $\mathcal{I}$-Set. I-Sets are used both as domains for $F D$ variables and as communication channels with an external source providing elements.

In this section, we briefly recall the syntax and semantics of the language.

In the following, we comply to the conventions in (Jaffar et al. 1998). In particular, every constraint domain $\mathcal{C}$ (where $\mathcal{C}$ can be $F D, \mathcal{I}$-Set, or $F D+\mathcal{I}$-Set) contains: the constraint domain signature $\Sigma_{\mathcal{C}}$, the class of constraints $\mathcal{L}_{\mathcal{C}}$ (a set of first-order $\Sigma$-formulas), the domain of computation $\mathcal{D}_{\mathcal{C}}$ (a $\Sigma$-structure that is the intended interpretation of constraints), the constraint theory $\mathcal{T}_{\mathcal{C}}$ (a $\Sigma$-theory that describes the logical semantics of the constraints), and the solver solv . 


\subsection{The $\mathcal{I}$-Set sort}

The $\mathcal{I}$-Set sort is meant to provide domains for variables in the $F D$ sort. Domains are thus considered as first-class objects, and they can be declaratively defined by means of constraints. In a sense, they can be considered as streams, but they are intrinsically non-ordered, and do not contain repeated elements. Declaratively, I-Sets are sets; thus unification and constraints should consider $\mathcal{I}$-Set terms modulo the set theory (Dovier et al. 1996): $\{A \mid\{A \mid B\}\}=\{A \mid B\},\{A \mid\{B \mid C\}\}=\{B \mid\{A \mid C\}\}$, which states that sets do not contain repeated elements and order is not important. Non-ground elements are forbidden in an I-Set; this restriction can be exploited for more efficient propagation algorithms. In fact, we represent an I-Set as the union of a set of ground elements (which we name the known part of the I-Set) and a variable representing its unknown part.

In $\operatorname{CLP}(\mathcal{I}$-Set $)$, the constraint domain signature, $\Sigma_{\mathcal{I} \text {-Set }}$, contains the following constraints:

- s-member $(E, S) \Leftrightarrow E \in S$,

- $\operatorname{union}(A, B, C) \Leftrightarrow A \cup B=C$,

- intersection $(A, B, C) \Leftrightarrow A \cap B=C$,

- $\operatorname{difference}(A, B, C) \Leftrightarrow A \backslash B=C$,

- $\operatorname{inclusion}(A, B) \Leftrightarrow A \subseteq B$,

where $E$ represents a ground term, and $A, B$, and $C$ represent I-Sets.

The operational semantics of the $\mathcal{I}$-Set sort is defined in terms of state of I-Sets, primitives used to check and modify the state, and events over I-Sets.

The state of an I-Set is defined by its known part, i.e. the set of the elements which are known to belong to the I-Set (as opposed to its unknown part, representing the elements which have not yet been acquired for the I-Set), and its open-closed condition, i.e., an I-Set is open if new elements can be inserted into it, closed otherwise.

A convenient notation to express the state of an I-Set is one based on Prolog-like lists. An I-Set is represented by a structure $S$ defined by $S::=\{\}$, or $S::=\{T \mid S\}$, or $S::=V$, where $T$ is a ground term and $V$ is a variable.

The known part of an I-Set is the set of all the ground elements in the list representing it; an I-Set is closed if its continuation (tail) is ground, open otherwise. For example, I-Set $\{1,2,3,4 \mid T\}$ is open, and its known part is the set $\{1,2,3,4\}$; I-Set $\{1,2,3,4\}$ has the same known part, but it is closed.

We have primitives to modify and check the state of an I-Set. Two primitives are used to modify the state, namely:

- ensure_member(Element,Iset): enforces Element to be a member of the known part of Iset, possibly adding it if it is not already member, or failing if it is not already member and the I-Set is closed;

- close(Iset): closes Iset; after execution of this primitive, no new elements can be added to the I-Set.

The following primitives are used to check the state of an I-Set: 
- known(Iset,KnownPart): KnownPart is the list of elements in the known part of Iset;

- is_closed(Iset): checks if the Iset is closed.

An event is a notification of how the status of the computation has been modified, which may be relevant for the rest of the computation and is to be processed properly. The semantics of an event is defined by rules specifying its interactions with the constraints in the store. It is quite apparent that the concept of event finds a natural representation as a CHR constraint; nevertheless, we prefer, in defining the operational semantics of the language, to keep events and proper $\mathcal{I}$-Set constraints distinct. The concept of event makes it possible to express the semantics of $\mathcal{I}$-Set constraints with simple (CHR-like) rules. For example, this rule is all that is needed to define the fact that, in the inclusion/2 constraint, all elements in the first I-Set also appear in the second:

$$
\begin{aligned}
& \text { inserted(Element, Iset } 1), \text { inclusion (Iset } 1, \text { Iset } 2) \Longrightarrow \\
& \text { ensure_member (Element, Iset } 2)
\end{aligned}
$$

This rule simply states that, if the inserted(Element,Iset1) event is raised (i.e., if Element has been inserted into Iset1) and Iset1 is known to be included in Iset2, the propagation process must make sure that the element is also present in Iset2. This may imply the insertion of Element into Iset2, with a subsequent inserted(Element,Iset2) event, if Iset2 is open and Element is not already a member, or a failure, if Iset2 is closed and Element is not already a member.

Propagation of closure can also be managed with ease. For instance, the rule

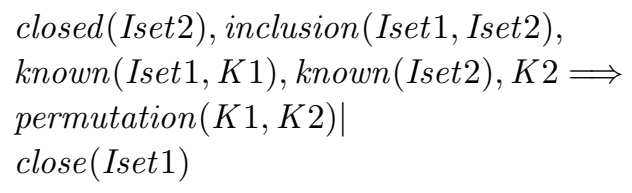

states that if Iset $1 \subseteq$ Iset 2, Iset 2 is closed (as indicated by the closed(Iset2) event), and the known elements in Iset 1 are all the known elements in Iset2, then also Iset 1 is closed (by the close(Iset1) primitive).

\subsection{The FD sort}

The $F D$ sort shares the same declarative semantics of the classical $F D$ sort. Thus, the usual constraints in CLP $(F D)$ are considered (arithmetic, relational constraints plus user-defined constraints). We suppose that the symbols $<, \leq,+,-, \times, \ldots$ belong to $\Sigma_{F D}$ and are interpreted as usual.

Since we want cope with incompletely specified variable domains avoiding useless value acquisition, we use a constraint propagation based on the available knowledge, when domains are still partially specified. For this reason, we proposed (Gavanelli et al. 2004) an extension, for the partially known case, of the concept of consistency, called known consistency. In this paper, we provide only the definition of node and arc-consistency; the extension to higher degrees of consistency is straightforward. 
Definition 2.1

A unary constraint $c\left(X_{i}\right)$ is known node-consistent iff

$$
\forall v_{i} \in K_{i}^{c}, v_{i} \in c\left(X_{i}\right),
$$

where $K_{i}^{c}$ is the known part of the domain of $X_{i}$. A binary constraint $c\left(X_{i}, X_{j}\right)$ is known arc-consistent iff

$$
\forall v_{i} \in K_{i}^{c}, \exists v_{j} \in K_{j}^{c} \text { s.t. }\left(v_{i}, v_{j}\right) \in c\left(X_{i}, X_{j}\right),
$$

where $K_{i}^{c}$ and $K_{j}^{c}$ are the known parts of the domains of $X_{i}$ and $X_{j}$, respectively. A constraint network is known arc-consistent $(K A C)$ iff all unary constraints are known node-consistent and all binary constraints are known arc-consistent.

The following proposition shows the link between known arc-consistency and arcconsistency (Mackworth 1977). The proof can be found in (Gavanelli et al. 2004).

\section{Proposition 1}

Every algorithm achieving KAC (i.e. any algorithm that computes an equivalent problem that is $\mathrm{KAC}$ ) and that ensures at least a known element in each variable domain is able to detect inconsistency in the same instances as an algorithm achieving AC.

In other words, if there exists an arc-consistent sub-domain, then there exists a maximal arc-consistent sub-domain; so if KAC does not detect inconsistency, AC will not detect inconsistency either.

$\mathrm{KAC}$ is equivalent to $\mathrm{AC}$ when domains are completely known. The advantage in using $\mathrm{KAC}$ is that the check for known arc-consistency can be performed lazily, without full knowledge of all the elements in every domain.

\subsection{Linking the two sorts}

Intuitively, we want to bind $\operatorname{CLP}(F D)$ with $\operatorname{CLP}(\mathcal{I}$-Set) with the intended semantics that I-Sets provide domains for $F D$ variables.

Given the two CLP languages $\mathcal{L}_{F D}$ and $\mathcal{L}_{\mathcal{I} \text {-Set }}$, we define the CLP language $\mathcal{L}$ as the union of the two languages, with a further constraint, :: , defined as follows:

- the signature $\Sigma=\Sigma_{F D} \cup \Sigma_{\text {I-Set }} \cup\{::\}$;

- the intended interpretation $\mathcal{D}$ keeps the original mappings in the $F D$ and $\mathcal{I}$-Set sorts; i.e., $\left.\mathcal{D}\right|_{\Sigma_{F D}}=\mathcal{D}_{F D}$ and $\left.\mathcal{D}\right|_{\Sigma_{\text {I-Set }}}=\mathcal{D}_{\text {I-Set }}$.

The declarative semantics of the constraint is

$$
X:: S \leftrightarrow X \in S
$$

where $X$ is a $F D$ variable. The :: /2 constraint links a $F D$ variable to its domain as in most $C L P(F D)$ frameworks, with the difference that $S$, being and I-Set, may be non-completely specified. The :: $/ 2$ constraint should not be confused with the s-member/2 constraint of Section 2.1 which represents the constraint of set membership between a ground element and an I-Set. 


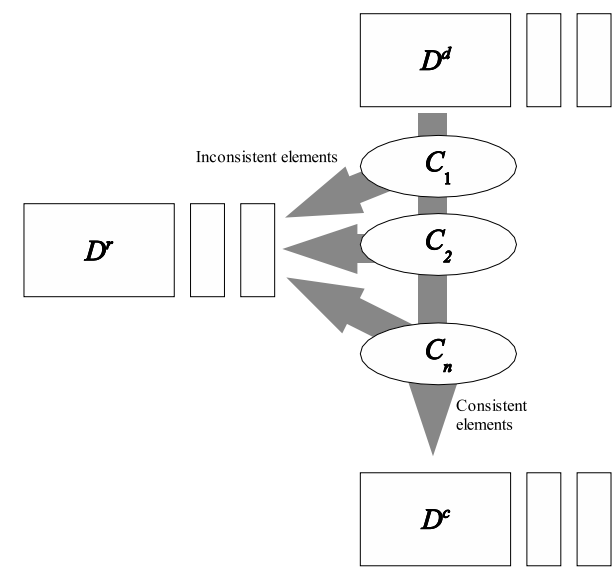

Fig. 1. FD Constraints as filters on variable domains

In CLP $(F D)$ systems, domains provide ancillary information about variables. Domains contain the possible values that a variable can take; if a value is not consistent with the imposed constraints, it is operationally removed from the domain. This helps many systems (Dincbas et al. 1988 Puget 1994 IC-Parc 2001) SICStus 2003) to obtain higher performance; in fact, domain wipe-outs are detected early and many alternatives are efficiently pruned. On the other hand, in the $\mathcal{I}$-Set sort, domains must be manipulated as logical entities: if an element declaratively belongs to a domain, it cannot be removed.

Suppose that we have constraint $\{1,2,3\} \subseteq D$ stating that the elements 1,2 and 3 should belong to the set $D$, the constraint $X:: D$ that links variable $\mathrm{X}$ to the domain $\mathrm{D}$, and $X \neq 1$ that states that $\mathrm{X}$ should be different from 1 . Usual constraint propagation of the constraint $X \neq 1$ would remove element 1 from the domain $D$, but this is inconsistent with the constraint $\{1,2,3\} \subseteq D$ so the computation would fail. This behavior is not correct, because the set of constraints $\{1,2,3\} \subseteq D$, $X \in D$, and $X \neq 1$ is satisfiable.

For this reason, in our framework, the domain of a variable $X$ is represented by two streams: a Definition Domain, $D_{X}^{d}$, that contains all the values synthesized for the variable, and a stream of Removed Values, $D_{X}^{r}$, that is the set of elements proven to be inconsistent with the imposed constraints. The set of available items for $X$, also called Current Domain, $D_{X}^{c}$, is given by the relation:

$$
D_{X}^{c}=D_{X}^{d} \backslash D_{X}^{r}
$$

It should be noticed that $D_{X}^{c}$ remains open until $D_{X}^{d}$ is closed. $D_{X}^{r}$, instead, is always open, so to make it possible to move elements into it from the current domain even if the definition domain has been closed.

With the imposed constraints, it is possible to declaratively add a newly synthesized value to the definition domain (imposing the constraint $v \in D_{X}^{d}$ ) or remove an inconsistent element from the current domain ( $w \notin D_{X}^{c}$ or, equivalently, $w \in D_{X}^{r}$; see Fig. (1). In this way, during search, an inconsistent element will not be tried if it belongs to $D_{X}^{r}$ and a possible domain wipe-out will be detected when $D_{X}^{c}$ is empty. 
Note that the relation in Eq. (2) automatically propagates two types of information from the definition domain to the current domain of a variable. First, whenever a domain element is synthesized, it is considered in the current domain and can be exploited for propagation. Second, if no more values are available to the definition domain, also the current domain becomes closed.

Notice that more than one FD variable can range on the same definition domain or, equivalently, their definition domains can be linked by an equality constraint; however, each of them will have its own current domain and set of removed values. By definition, the user cannot close the current domain of a variable: the user should only access the definition domain directly. This is not a restriction, because if one wants to close independently the current domain of different variables (as in the previous example), he can define two different definition domains (and, possibly, impose some constraints among them, e.g., $D_{X}^{d} \subseteq D_{Y}^{d}$ ).

In order to achieve $\mathrm{KAC}$, it is necessary to remove elements and to promote elements, i.e., to move ideally some elements from the unknown part to the known part. Elements can then be removed (i.e., prevented from entering the current domain) if they are shown to be inconsistent. An algorithm for achieving KAC is shown in (Gavanelli et al. 2004).

The addition, besides the deletion, of elements to the domain might seem non monotonic. However, the current domain is kept open as long as new acquisitions are possible (i.e., until the definition domain is closed), the unknown part representing the set of future acquisitions. Thus, declaratively, when a new element enters the known part of the current domain, it is not properly added: it is just made explicit. A similar behavior is also achieved by Mailharro (1998) with the use of a wildcard representing values entering the domain.

Clearly, the repeated acquisition of the same element would result in a loop; this can be avoided by having an acquisition module that does not provide twice the same element to the same I-Set (as hypothesized, for instance, by Mailharro (1998)).

Example: Numeric CSP. Various applications could be thought exploiting interactive constraint propagation, as many systems need to interact with an external environment during propagation: some real-world examples can be found in (Gavanelli et al. 2004). In this section, we give a simple example, aimed at showing a typical ICSP computation, rather than at showing the power of the language.

With the given language, we can state in a natural way the following problem:

$$
:-X:: D_{X}, Y:: D_{Y}, Z:: D_{Z} \text {, intersection }\left(D_{X}, D_{Y}, D_{Z}\right), Z>X \text {. }
$$

defining three variables, $X, Y$, and $Z$, with constraints on them and their domains. KAC propagation can start even with domains fully unknown, i.e., when $D_{X}, D_{Y}$ and $D_{Z}$ are variables. Let us suppose that the elements are acquired through interaction with a user and the first element retrieved for $X$ is 1 . This element is inserted in the definition domain of $X$, i.e., $D_{X}^{d}=\left\{1 \mid\left(D_{X}^{d}\right)^{\prime}\right\}$; since it is consistent with FD constraints, it is not redirected to the $D_{X}^{r}$ stream; value 1 is considered in the current domain, i.e., $D_{X}^{c}=\left\{1 \mid\left(D_{X}^{c}\right)^{\prime}\right\}$. Then KAC propagation tries to find 
a support for this element in each domain of those variables linked by $F D$ constraints; in our instance $D_{Z}$. A value is requested for $D_{Z}$ and the user gives a (possibly consistent) value: 2 . This element is inserted into the definition domain of $Z: D_{Z}^{d}=\left\{2 \mid\left(D_{Z}^{d}\right)^{\prime}\right\}$. The constraint imposed on domains can propagate, so element 2 has to be inserted in the definition domain of $Y$ and $X$, thus $D_{Y}^{c}=D_{Y}^{d}=\left\{2 \mid D_{Y}^{\prime}\right\}$ and $D_{X}^{c}=D_{X}^{d}=\left\{1,2 \mid D_{X}^{\prime \prime}\right\}$.

Since the acquired element is consistent with FD constraints involving $Z$, it enters the current domain of $Z$. KAC propagation must now find a known support for element 2 for $X$, so another request is performed. If the user replies that there is no other element in the domain of $\mathrm{Z}$, then 2 is sent to the stream of removed values for $X$ (thus, it is not considered in the current domain of $X$ ). It will remain in the definition domain because the element semantically belongs to the domain even if no consistent solution can exist containing it.

When KAC propagation reaches the quiescence, each element of the current domain of each variable has a support for each FD constraint involving that variable.

\section{Implementation concepts}

Since one of the aims of the ICSP model is to manage efficiently those problems in which value acquisition is costly, it is useful to exploit the link between the two sorts to avoid unnecessary acquisition of domain elements. For instance, as shown in the example in Section 2.3 it is possible to infer the presence of a value in the known part of an I-Set from $\mathcal{I}$-Set constraints, without acquiring it directly.

Thus, for the sake of efficiency, the implementation of the language must be aware of the link between the two sorts, given by the :: /2 constraint (see Section 2.3], and exploit it when useful. Nonetheless, the two sorts are clearly distinct, and need to be handled by different computational mechanisms.

In more detail, it is possible to devise two constraint solvers: one for the $F D$ sort, meant to ensure KAC (see Def. 2.1) of the FD constraint network, and one for the $\mathcal{I}$-Set sort, meant to ensure satisfaction of $\mathcal{I}$-Set constraints.

These solvers only need to interact in two cases:

- when, during $F D$ KAC check, a new element is acquired, $\mathcal{I}$-Set propagation must be activated in order to satisfy $\mathcal{I}$-Set constraints;

- conversely, when a new element enters the known part of an I-Set, FD KAC check must be activated over all the $F D$ variables which have their definition domain in the I-Set.

The CHR language is a perfectly suitable tool for this purpose, letting us deal with the two sorts separately, and to manage the link between them with ease.

\subsection{FD sort concepts}

The aim of the $F D$ solver is to keep the constraint network known arc-consistent. This is achieved by preventing elements from entering current domains if no KAC support is found for the constraints involving them. In more detail, each time a new 


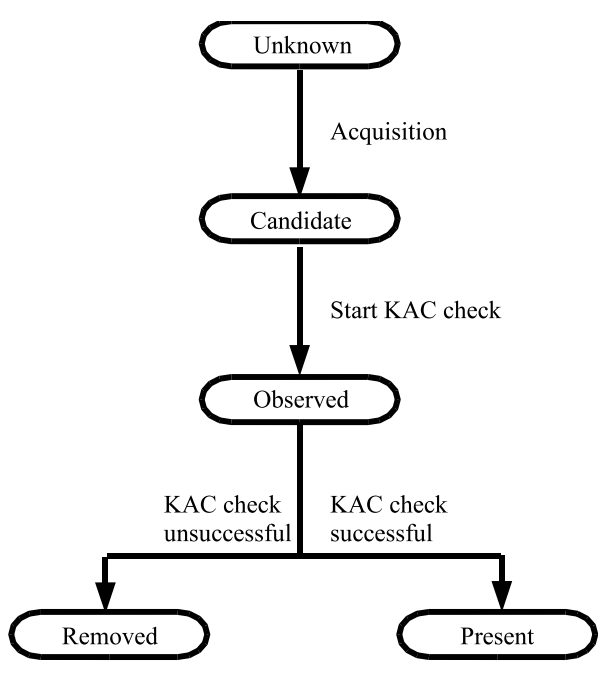

Fig. 2. State transitions for (Variable,Element) pairs

element $E$ is promoted from the unknown to the known part of an I-Set $C$, it is inserted into the definition domain of each variable $V$ such that $V:: C\left(E \in D_{V}^{d}\right.$ with the notation of Sect. 2.3). The algorithm then tries to find a KAC support for $E$ : if support is found, then $E$ is also inserted into the Current Domain of $V$ $\left(E \in D_{V}^{c}\right.$ ); otherwise, although it stays in the definition domain of $V$, it enters the stream of Removed Values for $V\left(E \in D_{V}^{r}\right)$.

In this way, at any step of the propagation, all of the values in current domains are certainly supported.

\subsubsection{Values' states}

The process described above can be clearly formalized as a sequence of state transitions for $(V, E)$ pairs. These states are:

- unknown: $E$ has not yet been acquired as a value for $V$;

- candidate: $E$ has been inserted into $D_{V}^{d}$, but $(V, E)$ has not yet been chosen for supporting another value and no KAC control is being run on it;

- observed: $(V, E)$ provides support for some other observed pair, but it has not yet been proven to be supported;

- present: $(V, E)$ is supported, and has thus been inserted into $D_{V}^{c}$;

- removed: $E$ is not supported, and has thus been inserted into $D_{V}^{r}$.

The possible state transitions are shown in Figure 2

\subsubsection{Lazy value acquisition}

The ICSP framework is more suitable for those applications in which the process of acquiring domain values is computationally costly. In this perspective, the KAC check procedure has been designed so as to acquire new values only when it is 
really necessary, i.e., when no support for a value can be found among already known values.

\subsection{I-Set sort concepts}

The task performed by the $\mathcal{I}$-Set solver is to ensure the satisfaction of the $\mathcal{I}$-Set sort constraints, and to propagate the closure of definition domains, when possible. As shown in the example below, it is possible to infer that an element belongs to an I-Set from $\mathcal{I}$-Set sort constraints, thus reducing the number of necessary acquisitions and improving efficiency.

In particular, each time an element is added to an I-Set, an inserted $/ 2$ event (see Section 2.11) is raised, which starts a check for the satisfaction of the $\mathcal{I}$-Set constraints involving the I-Set itself.

Example Let us suppose the constraint intersection $\left(D_{X}, D_{Y}, D_{Z}\right)$ is given among the $D_{X}, D_{Y}$ and $D_{Z}$ I-Sets, and that the current values of the domains are

$D_{X}=\left\{2,4 \mid D_{X}^{\prime}\right\} ; D_{Y}=\left\{3,4 \mid D_{Y}^{\prime}\right\} ; D_{Z}=\left\{4 \mid D_{Z}^{\prime}\right\}$.

If 5 is added to $D_{Z}$, the system immediately ensures 5 to be a member of both $D_{X}$ and $D_{Y}$, without the need for acquiring the element. If 3 is added to $D_{Z}$, the system only needs to add it to $D_{X}$. Conversely, if 3 is added to $D_{X}$, it is also added to $D_{Z}$; if 1 is added to $D_{X}$, instead, the system cannot infer anything, and thus makes no additions.

$\mathcal{I}$-Set constraints also have to be taken into account to propagate closure of I-Sets, when possible (as in the example shown in Sect. 2.11). For this purpose, we use a closed/1 event, which notifies that an I-Set has been closed.

\subsection{Interaction between sorts}

The link between the two sorts, represented by the :: /2 constraint (see Section 2.3), is implemented as an interaction mechanism between the solvers activated when, on the one hand, a new element is acquired during a KAC check and, on the other hand, an element is inserted into an I-Set.

Semantically, we use the inserted/2 event for both of these cases (see Section 2.1).

At the implementation level, the event causes the control to pass from one solver to another.

\subsection{Algorithms}

\subsubsection{Priority among supporting values}

A (Variable,Element) pair is supported if, for each constraint involving Variable, support is found in the known part of other variables' domains. But supporting pairs may not be supported in their turn: this implies that, if a pair is found to be unsupported, new support needs to be found for all of the pairs that it was supporting. For efficiency, we keep track of which pairs support which other pairs 
for which constraints, so that, in case a pair is found unsupported, it is possible to revise only the affected constraints.

However, because of states (see Sect. 3.1.1), it is possible to reduce the number of supports to keep track of. If an element is supported by an element whose state is present, there is no need for keeping track of the support, because a present element is already known to be supported, as explained in Sect. 3.1.1 Thus, when seeking support for a constraint, present elements should be tried first.

Likewise, an element whose state is observed is more convenient than an element whose state is candidate, because the algorithm is already seeking support for it, and can thus avoid seeking support for a new element (note that all candidates will eventually be checked; but in this way, when their turn comes, there will be, in general, more present elements).

So, a priority can be established among eligible supporting elements: first present, then observed, then candidates. Only when no support is found among these elements, a new element needs to be acquired: if the definition domain of the corresponding variable is open, an element can be requested, otherwise the support seek procedure fails.

\subsubsection{The support graph}

Support dependencies can be represented by a (directed) support graph, in which nodes represent observed (Variable,Element) pairs, and arcs represent support dependencies.

When a candidate pair becomes observed, the corresponding node is added to the graph. This may happen at the beginning of graph construction, when the graph is empty and the candidate is chosen to be the first node, or later, during the support seeking procedure, if the candidate provides support for an observed.

A new node needs support for the $F D$ constraints involving it. For each $F D$ constraint, the following attempts, in this order (see Sect. 3.4.1) are made, until one succeeds:

1. support is found in present elements only: the graph needs no modifications;

2. one of the supporting elements is observed: an arc is added from the supporting node to the supported, marked with the constraint;

3. one of the supporting elements is candidate: a new node is added for the candidate, an arc is added from the supporting node to the supported, marked with the constraint.

If none of these attempts succeeds, a variable (other than Variable) is chosen for acquisition among those involved by the constraint, and the search for support is restarted from the support-in-candidates attempt (step 3). The search for support needs to be restarted because, in order to satisfy $\mathcal{I}$-Set constraints, new candidates may have been added, which may provide support for the considered $F D$ constraint.

A node is deleted from the graph if it is found to have no support; in this case, new support is sought for all of the nodes that it was supporting.

Once a graph is fully built, all of its nodes can be inserted into the current 
domains (observed-to-present transition), whereas the unsupported nodes will be inserted into the stream of removed values.

The procedure reaches quiescence only when there are no more candidates to search support for.

\section{CHR implementation}

In this section, we show how the concepts explained in Section 3 have been implemented in a constraint solver using the Constraint Handling Rules library of SICStus Prolog.

\subsection{I-Set variables and constraints}

I-Sets are represented by CHR variables: these variables (also "link variables" hereafter) act as a link for the information stored in constraints, and are never instantiated. To keep memory of the state of I-Sets we use CHR constraints, meant to interact in order to ensure satisfaction of $\mathcal{I}$-Set constraints.

The state of an I-Set is represented by means of three CHR constraints: iset_known(Iset,Known) links an I-Set to its known part, iset_open(Iset) indicates that the I-Set is open (i.e., other elements can be inserted), and closed(Iset) indicates that the I-Set is closed.

An I-Set is created by the user either explicitly (new_iset_object/3 predicate) or implicitly, imposing constraint icsp_def_domain/2 (which implements the ::/2 constraint of Sect. 2.3) between an $F D$ variable and the link variable of an I-Set. It is possible to define the domain as empty or as having a starting known part.

Each constraint among I-Sets (see Section 2.1 for the formal definitions) is represented by a corresponding CHR constraint among the link variables of the I-Sets that it involves, namely the iset_member/2 (in this case, the first argument is a ground $F D$ value), iset_union $/ 3$, iset_intersection $/ 3$, iset_inclusion $/ 2$, and iset_difference/3 constraints.

The satisfaction of $\mathcal{I}$-Set constraints is ensured incrementally (see Section 3.2). For instance, the following rule is used for implementing the iset_intersection/3 constraint.

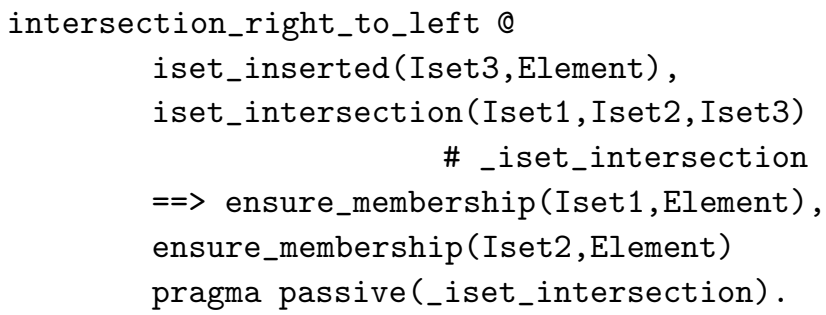

The rule is activated when a new element is added to the I-Set represented by the third argument of an iset_intersection/3 constraint: this is notified by the iset_inserted/2 constraint, which implements the inserted event of Sect. 2.1 The rule imposes constraint ensure_membership/2 on each of the other two I-Sets and 
the element; if necessary, the element will be inserted into the I-Sets. Notice that ensure_membership/2 is defined so as to fail if the I-Set is closed and the element is not already in its known part. Constraint iset_intersection/3 is declared passive for efficiency, because it is not necessary to generate code for it $(\mathcal{I}$-Set constraints as iset_intersection $/ 3$ are imposed only at the beginning of the computation).

Two more rules (not shown here for lack of space) manage the case of an element having been inserted into the first or second argument of the iset_intersection/3 constraint.

The close/1 primitive of Sect. 2.1] is implemented by the following CHR:

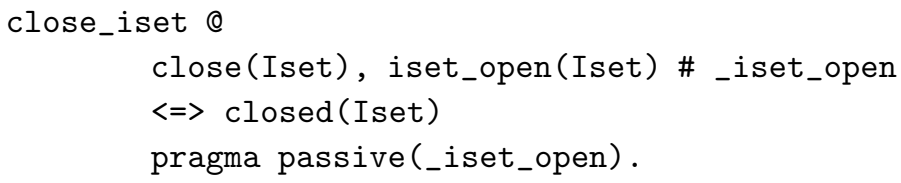

CHR constraint close/ 1 is imposed to close the I-Set; the effect of this rule is to remove constraint iset_open/1 for the I-Set, and to impose CHR constraint closed/1 for the I-Set. Constraint closed/1 also implements the event notifying that the argument I-Set has been closed, which can interact with $\mathcal{I}$-Set constraints so as to propagate closure when possible. For instance, the rule of Sect. 2.1 is implemented as follows:

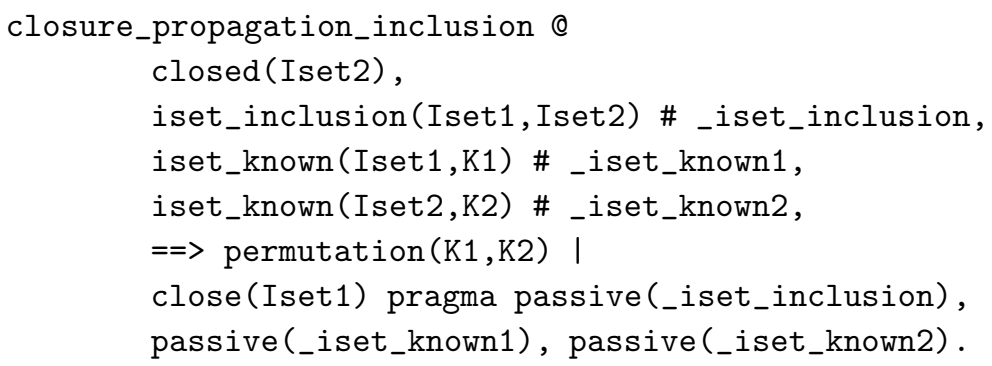

Predicate permutation $/ 2$ in the guard checks that the two I-Sets have the same elements.

\subsection{FD variables and constraints}

\subsubsection{FD variables and domains}

$F D$ variables are represented as CHR constrained variables. Their domain is an I-Set; constraint ::/2 (see Section 2.3) is implemented as the icsp_def_domain(Variable,Iset) CHR constraint, whereas the current domain of the variable and its set of removed values are represented by the icsp_curr_domain(Variable,(Present,Removed)) CHR constraint, where Present is the list of present elements and Removed is the list of removed elements.

\subsubsection{FD constraints}

$F D$ constraints are represented by the fd_constraint(ConstraintName, ListOfArgu- 
ments) CHR constraint. For instance, $F D$ constraint $X<Z$ is represented by the fd_constraint (lt, [X, Z] ) CHR constraint.

$F D$ constraints are defined by the user simply as one or more clauses for the Prolog predicate $f d_{-}$verify/2 needed to verify whether the constraint is satisfied by a list of ground arguments. This way of defining $F D$ constraints supports non-binary constraints, and easy extensibility of the system.

For instance, a possible definition of the $F D</ 2$ constraint could be

fd_verify $(1 t,[A, B]):-A<B$.

\subsubsection{KAC procedure}

Candidate (see Section 3.1.1) elements for each variable are stored in the current_candidates(Variable,ListOfElements) constraint.

KAC check over candidates is performed by building a support graph (see Section 3.4.2). Nodes of the graph are (Variable, Element) pairs. A pair is a node of the graph if and only if it is member of the list argument of the observed_candidates/1 CHR constraint; an arc (indicating support dependency) is represented by the relies ((Var1,Val1), (Var2, Val2), FDConstraint) CHR constraint, where FDConstraint is the $F D$ constraint for which (Var2, Val2) supports (Var1, Val1).

Graph construction is achieved by the following CHR rule:

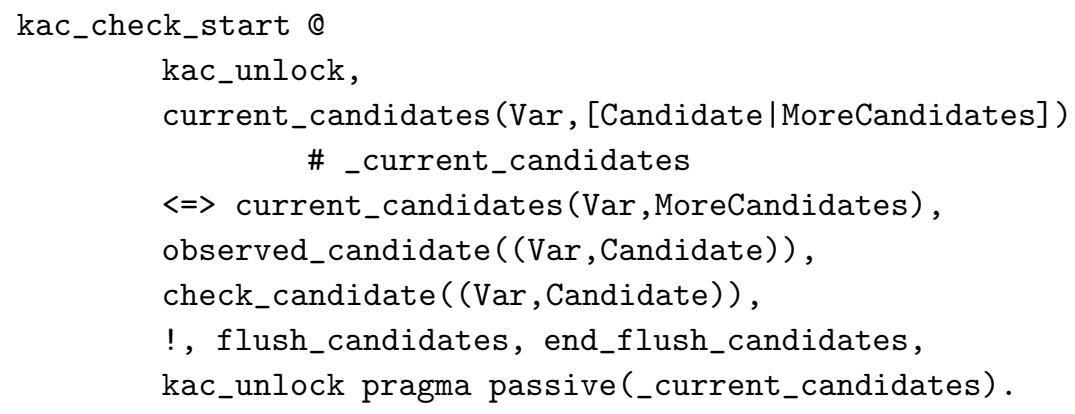

Graph construction begins when there is at least one non-empty list of candidates for a variable: an element is chosen (in the current implementation, it is simply the first of the list), pair (Variable, Element) becomes the first node of the graph (constraint observed_candidate/1 adds its argument to the list argument of constraint observed_candidates/1), and KAC check starts from it (check_candidate/2 constraint); during check, new nodes may be added to the graph, which will be checked for support in their turn. When the construction is done, graph nodes are inserted into the current domains (flush_candidates/0 and end_flush_candidates/0 constraints), and the procedure can start again over the rest of candidates.

kac_unlock/0 is a constraint meant simply to start the KAC procedure. If there are no candidates when it is imposed, then if there is a variable with empty current domain an element is acquired for it which will become a candidate; otherwise, the procedure reaches quiescence. 
Support seek for a (Variable, Element) pair is started by constraint check_candidate((Variable,Element)). This constraint collects from the store all the FD constraints that involve Variable, and for each of them tries to find an assignment of all the involved variables that satisfies it (i.e., that makes goal $f d \_v e r i f y / 2$ (see Section 4.2.2) succeed). Elements for the other variables are chosen following the priority described in Section 3.4 .1

\subsubsection{Support seek through element acquisition}

If no acceptable assignment is found among known, observed, and candidate elements, then:

- if the I-Set domain of at least one of the other variables is open, a new element is acquired for one of the other variables;

- if the I-Set domains of all the other variables are closed, failure is reported, and possibly backtracking is applied.

Which variable should be chosen for acquisition among those with open domain is not obvious for non-binary constraints, and application specific heuristics could be useful; in this implementation the first variable is simply chosen.

In the current system, element acquisition is obtained by asking the user for an element, and the I-Set is closed in case of a predefined input from the user. Obviously, in practical applications, elements would be provided by an acquisition system, as in (Mailharro 1998), where the acquisition is done automatically by generation of new component instances, or in (Cucchiara et al. 1999b), where elements are provided by a low-level segmentation system.

\subsection{Constraints and rules for interaction between sorts}

Only one CHR constraint needs to be added to the solver to link the two sorts: iset_inserted(Iset,Element), imposed when Element is inserted into the known part

of Iset. This constraint is the implementation of the inserted/2 event described in Section 2.1]

\subsubsection{FD to $\mathcal{I}$-Set link}

When a new element Element is acquired for a variable $V$ whose definition domain is Iset, iset_inserted(Iset,Element) is imposed. This CHR constraint implements an event that triggers the $\mathcal{I}$-Set constraint check. From a procedural point of view, this makes control pass from the $F D$ solver to the $\mathcal{I}$-Set solver.

\subsubsection{I-Set to FD link}

When imposed, iset_inserted(Iset,Element): 
- first, interacts with the $\mathcal{I}$-Set constraints involving Iset, as shown in Section 4.1

- then, inserts Element in the list of candidates of all the variables having Iset as definition domain, for later support seek.

The interaction with the $\mathcal{I}$-Set constraints may involve new insertions, which will, in their turn, impose further iset_inserted/2 constraints.

New candidates are added only when $\mathcal{I}$-Set propagation has been completed, for the reasons explained in Section 3.2

\section{Related work}

I-Sets can be considered as streams with a set semantics (i.e., in an I-Set there are no repeated elements and elements are not sorted). Streams are widely used for communication purposes in concurrent logic programming (Shapiro 1987). Various communication protocols can be implemented using this simple yet powerful data structure (Shapiro 1989). An I-Set can only contain ground elements; this restriction prevents (open) I-Sets from being passed in an I-Set, but lets us achieve higher efficiency.

The first results of our research in the ICSP framework are reported by Cucchiara et al. (1999a), where the ICSP model is proposed as an extension of the CSP model. In this model, variables range on partially known domains which have a known part and an unknown part represented as a variable. Domain values are provided by an extraction module and the acquisition process is (possibly) driven by constraints. The model has been proven effective in a vision system (Cucchiara et al. 1999b), in randomly-generated problems (Cucchiara et al. 1999a), and in planning (Barruffi et al. 1999). This work can be considered as the language extension and CHR implementation of the ICSP framework, maintaining it as the core of the propagation engine on the $F D$ side.

Operationally, achieving KAC has some similarities with achieving Lazy Arc Consistency (LAC) (Schiex et al. 1996). LAC is an algorithm that finds an arcconsistent sub-domain (not necessarily a maximal one) and tries to avoid the check for consistency of all the elements in every domain. KAC looks for an arc-consistent sub-domain as well, but it is aimed at avoiding unnecessary information retrieval, rather than unnecessary constraint checks.

Codognet and Diaz (1996) describe a method for compiling constraints in $\operatorname{CLP}(F D)$. There is only one primitive constraint ( $X$ in $R$ ), used to implement all the other constraints. $R$ represents a collection of objects and can also be a user function. Thus, in CLP $(F D)$ domains are managed as first-class objects; our framework can be fruitfully implemented in systems exploiting this idea.

Sergot (1983) proposes a framework to deal with interaction with the user in a logic programming environment. Our work can be used for interaction in a CLP framework; it lets the user interactively provide domain values.

Zweben and Eskey (1989) propose an algorithm that evaluates domain elements only when necessary; domains are streams and constraints are filters on domains. 
Dent and Mercer (1994) show the effectiveness of such an approach when constraint checks are expensive operations. In our proposal, implementation of delayed evaluation is quite natural and simple, even if our work is aimed at reducing domain values extractions, not constraint checks.

Dynamic Constraint Satisfaction (DCS) (Dechter and Dechter 1988) is a promising field of AI taking into account dynamic changes of the constraint store such as the addition and deletion of values and constraints. The difference between DCS and our approach concerns the way of handling these changes. DCS approaches propagate constraints as if they worked in a closed world. Basically, in a DCS one can add or remove a constraint; thus, one can also add and delete domain elements provided that they are all known from the beginning. In an ICSP, instead, domain elements that are unknown can be requested and inserted in the domain. DCS solvers record dependencies between constraints and the corresponding propagation in proper data structures (Schiex and Verfaillie 1993) so as to tackle modifications of the constraint store as soon as data change. In this perspective, we also cope with changes since the acquisition of new values can be seen as a modification of the constraint store. However, we work in an open world where domains are left open thanks to their unknown part. Unknown domain parts represent intensionally future acquisitions, i.e., future changes.

Another DCS framework was given for configuration problems (Mittal and Falkenhainer 1990). This framework considers dynamicity in the set of variables; variables are introduced or removed during search by means of constraints. The aim is to find a solution where only some of the variables are assigned a value, while the others are inactive. However, differently from our framework, the set of domain values is given at the specification of the problem.

Many systems consider implement sets, because sets have powerful description capabilities. In particular, some have been described as instances of the general $\operatorname{CLP}(\mathcal{X})$ framework (Jaffar et al. 1998), like $\{\log \}$ (Dovier and Rossi 1993, Dovier et al. 1996 Dovier et al. 2001), CLPS (Legeard and Legros 1991), or Conjunto (Gervet 1997). Others apply an object-oriented approach (Puget 1992, Puget 1994).

In $\{\log \}$ (Dovier and Rossi 1993, Dovier et al. 1996, Dovier et al. 2001), a set can be either the empty set $\emptyset$, or defined by a constructor with which, given a set $S$ and an element $e$, returns the set composed of $S \cup\{e\}$. This language is very powerful, allowing sets and variables to belong to sets. However, set unification and the propagation of other constraints has an exponential time complexity in the worst case. If we allow non-ground elements in an I-Set, we obtain a more expressive (although less efficient) framework, like $\{\log \}$; we are currently studying this extension.

Set variables (Puget 1992 Puget 1994) can range on set domains. Each domain is represented by its greatest lower bound and its least upper bound. Each element in a set must be ground, sets are finite, and they cannot contain sets. These restrictions avoid the non-deterministic unification algorithm, and give good performance results; they are implemented in most Constraint Programming systems (Puget 1994. Smolka 1995 SICStus 2003, Gervet 1997 IC-Parc 2001). However, these systems do not deal with problems in which some domain elements are not known; in fact, 
the user must provide the least upper bound of each set, thus giving the universe set at the beginning of the computation.

ILOG-Solver (Puget 1994) does not deal directly with those problems, but has an extension module, called ILOG-Configurator (Mailharro 1998, ILOG 1999) whose main added value is to implement open domains in a way similar to our system. This system is very related with ours, thus we give a more detailed comparison.

Comparison with ILOG-Configurator. In Ilog-Configurator, there are variables called "ports" whose domains are defined by the set of instances of a given component type. The set of instances is not known in advance and instances are generated on demand during constraint propagation. In this way, domains of port variables are dynamically extended; a domain that can be extended contains an element called "wildcard".

Our system has many points in common with the one described by Mailharro (1998). To compare the two, the reader can refer to the following table:

ICSP ILOG-Configurator

\begin{tabular}{ll}
\hline \hline FD Variables & Port Variables \\
I-Set & Component Types \\
Known Part & Set of generated instances \\
Unknown Part & Set of not yet generated instances (Wildcard) \\
$:: / 2$ & Link port-type \\
Definition Domain $D_{X}^{d}$ & Set of instances of the target type \\
Current Domain $D_{X}^{c}$ & Possible set of a port \\
Removed Elements $D_{X}^{r}$ & Instances set of the target type $\backslash$ possible set \\
Inserted Event & Extension delta domain of ports \\
\hline
\end{tabular}

The differences that we envisage between the two systems are the following. ILOG-Configurator is based on an object-oriented technology, while ours is based on logic programming; this is reflected in some specific choices made in the two systems. For example, both the systems reason about the possible closure of a domain. This information is carried by a special element, called "wildcard" by Mailharro (1998), while in our system it is the unknown part of the domain. In our system, if the continuation of the domain is a variable, other elements can be added, otherwise, if it is the empty set, the insertion of other elements will be forbidden by unification. This has a declarative meaning from a set viewpoint; while a set containing a wildcard must be updated through some destructive assignment, our formalization lets the user specify the value of a logical variable: the continuation of the domain will be extended, as in a stream. In other words, in the ICSP formulation we keep set membership and set inclusion distinct. In fact, in (Mailharro 1998), the wildcard element represents a set of elements, but is also an element of the domain. Thanks to our formalization, some future extensions are possible: for example, it is possible to extend the type of sets in a domain, and to have domains that can 
contain sets themselves, like in $\{\log \}$ (Dovier et al. 1996). The propagation of FD constraints is operationally performed in a similar way (Known Arc Consistency propagation); however, the property of Known Arc Consistency (KAC), which was not defined by Mailharro (1998), enabled us to prove properties of the algorithms achieving KAC (Gavanelli et al. 2004).

Finally, in our framework it is possible to define I-Sets as the combination of other I-Sets with set operators.

\section{Conclusions}

In this work, we presented the implementation of a language that performs constraint propagation on variables with finite domains when information about domains is not fully known, and its CHR implementation. Domains are channels of information, and are considered as first-class objects that can be themselves defined by means of constraints. The obtained language belongs to the CLP class and deals with two sorts: the $F D$ sort on finite domains and the $\mathcal{I}$-Set sort for domains. We provide a propagation engine for the $F D$ sort exploiting known arc-consistency, and one for the $\mathcal{I}$-Set sort, as well as a mechanism for their interaction.

The source code of the system is available on request.

\section{Acknowledgements}

This work is partially funded by the Information Society Technologies programme of the European Commission under the IST-2001-32530 project (SOCS) within the Global Computing initiative.

We wish to thank the anonymous reviewers for their useful comments.

\section{References}

Barruffi, R., Lamma, E., Mello, P., and Milano, M. 1999. Least commitment on variable binding in presence of incomplete knowledge. In Proceedings of the European Conference on Planning (ECP99), S. Biundo and M. Fox, Eds. Lecture Notes in Computer Science, vol. 1809. Springer, Durham, UK, 159-171.

Codognet, P. And Diaz, D. 1996. Compiling constraints in clp(FD). Journal of Logic Programming 27, 3 (June), 185-226.

Cucchiara, R., Gavanelli, M., Lamma, E., Mello, P., Milano, M., and Piccardi, M. 1999a. Constraint propagation and value acquisition: why we should do it interactively. In Proceedings of the Sixteenth International Joint Conference on Artificial Intelligence, T. Dean, Ed. Morgan Kaufmann, Stockholm, Sweden, 468-477.

Cucchiara, R., Gavanelli, M., Lamma, E., Mello, P., Milano, M., and Piccardi, M. 1999b. Extending CLP(FD) with interactive data acquisition for 3D visual object recognition. In Proceedings of the First International Conference on the Practical Application of Constraint Technologies and Logic Programming. Practical Application Company, London, 137-155.

Cucchiara, R., Gavanelli, M., Lamma, E., Mello, P., Milano, M., and Piccardi, M. 2001. From eager to lazy constrained data acquisition: A general framework. New Generation Computing 19, 4 (Aug), 339-367. 
Dechter, R. AND DechteR, A. 1988. Belief maintenance in dynamic constraint networks. In Proceedings of the 7th National Conference on Artificial Intelligence, T. M. Smith, Reid G.; Mitchell, Ed. Morgan Kaufmann, St. Paul, MN, 37-42.

Dent, M. And Mercer, R. 1994. Minimal forward checking. In Proceedings of the Sixth International Conference on Tools with Artificial Intelligence. 432-438.

Dincbas, M., Van Hentenryck, P., Simonis, H., Aggoun, A., Graf, T., And Berthier, F. 1988. The constraint logic programming language CHIP. In Proceedings of the International Conference on Fifth Generation Computer System. OHMSHA Ltd. Tokyo and Springer-Verlag, Tokyo, Japan, 693-702.

Dovier, A., Omodeo, E., Pontelli, E., And Rossi, G. 1996. $\{\log \}$ : A language for programming in logic with finite sets. Journal of Logic Programming 28(1), 1-44.

Dovier, A., Pontelli, E., And Rossi, G. 2001. Constructive negation and constraint logic programming with sets. New Generation Computing 19, 3 (May), 209-256.

Dovier, A. And Rossi, G. 1993. Embedding extensional finite sets in CLP. In Proceedings of the 1993 International Symposium on Logic Programming. MIT Press, British Columbia, Canada, 540-556.

Faltings, B. And Macho-Gonzalez, S. 2003. Open constraint optimization. In Proceedings of the 9th International Conference on Principles and Practice of Constraint Programming (CP-2003). Lecture Notes in Computer Science. Springer, 303-317.

Frühwirth, T. 1998. Theory and practice of constraint handling rules. Journal of Logic Programming 37, 1-3 (Oct.), 95-138.

Gavanelli, M., Lamma, E., Mello, P., And Milano, M. 2004. Dealing with incomplete knowledge on CLP $(F D)$ variable domains. ACM Transactions on Programming Languages and Systems. To appear.

Gervet, C. 1997. Propagation to reason about sets: Definition and implementation of a practical language. Constraints 1, 191-244.

IC-PARC. 2001. ECL ${ }^{i} P S^{e}$ User Manual, Release 5.2. IC-Parc, Imperial College, London, $\mathrm{UK}$.

ILOG 1999. ILOG-Configurator, user manual, 1.0 ed. ILOG.

Jaffar, J., Maher, M., Marriott, K., And Stuckey, P. 1998. The semantics of constraint logic programs. Journal of Logic Programming 37(1-3), 1-46.

Legeard, B. And Legros, E. 1991. Short overview of the CLPS system. In Proceedings of the 3rd Int. Symposium on Programming Language Implementation and Logic Programming, PLILP91, J. Małuszyński and M. Wirsing, Eds. Lecture Notes in Computer Science. Springer-Verlag, Passau, Germany, 431-433.

Mackworth, A. 1977. Consistency in networks of relations. Artificial Intelligence 8, 99-118.

MailharRo, D. 1998. A classification and constraint-based framework for configuration. Artificial Intelligence for Engineering Design, Analysis and Manufacturing 12, 383-397.

Mittal, S. And Falkenhainer, B. 1990. Dynamic constraint satisfaction problems. In Proc. of AAAI-90. Boston, MA, 25-32.

Puget, J. 1992. PECOS: A high level constraint programming language. In Proceedings of the First Singapore International Conference on Intelligent Systems (SPICIS). Singapore, 137-142.

Puget, J. 1994. A C++ implementation of CLP. Tech. Rep. 94-01, ILOG Headquarters.

Schiex, T., Régin, J., Gaspin, C., And Verfaillie, G. 1996. Lazy arc consistency. In Proceedings of the Thirteenth National Conference on Artificial Intelligence and the Eighth Innovative Applications of Artificial Intelligence Conference. AAAI Press / MIT Press, Menlo Park, 216-221. 
Schiex, T. AND Verfaillie, G. 1993. Nogood recording for static and dynamic constraint satisfaction problems. In Proceedings of the 5th International Conference on Tools with Artificial Intelligence. IEEE Computer Society Press, Los Alamitos, CA, USA, 48-55.

Sergot, M. 1983. A query-the-user facility for logic programming. In Integrated Interactive Computing Systems, P. Degano and E. Sandewall, Eds. North-Holland, 27-41.

Shapiro, E., Ed. 1987. Concurrent Prolog - Vol. I. MIT Press.

Shapiro, E. 1989. The family of concurrent logic programming languages. ACM Computing Surveys 21, 4 (Mar.), 413-510.

SICStus 2003. SICStus Prolog user manual, release 3.11.0. http://www.sics.se/isl/sicstus/

SmolkA, G. 1995. The Oz programming model. In Computer Science Today, J. van Leeuwen, Ed. Lecture Notes in Computer Science, vol. 1000. Springer-Verlag, Berlin, 324-343.

Zweben, M. And Eskey, M. 1989. Constraint satisfaction with delayed evaluation. In IJCAI 89. Morgan Kaufmann, Detroit, 875-880. 